\title{
Introduction: Indigenous Knowledge in Development
}

\section{Paul Sillitoe}

\begin{abstract}
This is an introduction to indigenous or local knowledge (IK) in development. After discussing problems of definition, various models to represent relations between, and structure enquiries into, different knowledge traditions are outlined, including the continuum and sphere representations. This discussion includes a summary of points that justify why agencies should seek better to incorporate consideration of local knowledge into development programmes; and sketches the several methodological issues that we have to address to take this work forwards. Finally, this introduction concludes with some comments on the work of the Durham Anthropology in Development (AID) group.
\end{abstract}

KEYwORDS: Indigenous Knowledge (IK), models, methodology

It is some two decades since certain commentators started to advocate that international development take the incorporation of indigenous or local knowledge and practices into programmes more seriously (Howes and Chambers 1979; Brokensha et al. 1980; Rhoades and Booth 1982; Richards 1985; Thrupp 1989; Chambers et al. 1989). Several of us have subsequently added our voices to the chorus (Kloppenburg 1991; Warren 1991; DeWalt 1994; Warren et al. 1995; Purcell 1998; Antweiler 1998; Sillitoe 1998a; Ellen et al. 2000; Shepherd 2004; Bentley and Baker 2005). In Durham we seek to take this work forwards in the Anthropology in Development (AID) Group, which focuses on local knowledge in development issues. The articles presented in this issue of Anthropology in Action illustrate some of our interests and concerns in the field of indigenous knowledge in development.

\section{Definition of Indigenous Knowledge}

Indigenous knowledge (IK) is any understanding rooted in local culture. It includes all knowledge held more or less collectively by a population that informs interpretation of things. It varies between regions. It comes from a range of sources, is a dynamic mix of past 'tradition' and present invention with a view to the future.

People in different regions have unique cultural traditions and histories, which critically shape their views of their environments, livelihood choices, health and illness, social behaviour and so on. Indigenous knowledge research seeks to incorporate these views more into development. The term, particularly the indigenous part, has been subject to politically correct debate ('how can one define indigenous in the modern cosmopolitan world?') and several alternatives have been proposed including 
local knowledge, traditional knowledge, people's knowledge, 'glocal' knowledge and citizen science, to name some. We use indigenous knowledge as the term largely heard in development discourse and, where acronyms abound, the inevitable shorthand $I K$.

Indigenous knowledge informs behaviour, learnt from birth onwards. It focuses on provincial interests and concerns, often communicated in foreign idioms and styles, which we understand to varying extents. It is the heritage of everyday life, based on experience, often tested over centuries of use, adapted to local conditions. Repetitive practice may typify its learning, it being equally skill as formally transmitted knowledge. It is understanding, evolved over generations, subject to continual revision. While it is ever-changing and modified by information from elsewhere, it maintains its distinctive character, subject to ongoing local, regional and global negotiation.

Indigenous knowledge exists nowhere as a totality, or in comprehensive annals or allencompassing abstractions, although it may achieve some coherence in cosmologies, rituals and symbolic discourse (which are notoriously difficult to access convincingly). While not replicating one another's knowledge, members of the same culture share an indeterminate amount, having a common history, values and so on. Although IK is more widely shared generally than specialized scientific knowledge, no one person or social group knows it all. Its distribution is uneven. There may be some clustering of certain knowledge within populations (e.g. by gender, age, specialist status etc., maybe reflecting political power).

Indigenous knowledge enquiries go back, strictly speaking, to the beginning of anthropology, but they are relatively recent in development, their emergence linked to farming systems and participatory approaches (Sillitoe 1998b). These are wide ranging, in the farming systems tradition (FAO 1989), while not confining research largely to professionals, privileging scientific analysis and limiting local involve-

ment. They seek to make other views heard, in the participatory tradition, while being aware that such methods may encourage shallow understanding, are not culturally neutral and are subject to manipulation, outsiders often using them to ventriloquize their intentions (Wright and Nelson 1995; Cooke and Kothari 2001).

\section{Indigeous Knowledge and International Science}

Indigenous knowledge is often contrasted with, and until recently has been undervalued in comparison to, international scientific knowledge. This can hinder collaboration between scientists working in development and local people. Indigenous knowledge relates to specific cultural contexts whereas science has universal theoretical aspirations (Table 1). Indigenous practice is more inductive, with a 'weak' model of the world (often largely unknown to development outsiders), whereas scientific practice is more deductive, with a 'strong' model and agreed methods of investigation. We cannot assume that the two will be congruent, rather we have to seek contrasts and parallels. We have to reconcile IK, which is broad-based, workaday understanding, with scientific knowledge, which comprises narrow, specialist understanding, in seeking to promote cross-culturally informed research into development problems.

Table 1: Contrasts between IK and scientific research

Indigenous Knowledge

Local (specific)

Community dynamics and power relations

Participation

(collaborative)

Process

This bipolar discrimination between indigenous and scientific knowledge is inadequate, if 
not misleading as to the relationship and distinction between the two. We are not talking about two tenuously connected knowledge traditions separated by a cultural gulf, but rather a spectrum of relations. We can conceive of these relations as comprising a continuum (Figure 1). At one end are locals with little formal education, whose knowledge is largely locally derived, and at the other are trained scientists, contending with interdisciplinary research. In between are various intergradations of 'local insider' and 'global outsider' knowledge. Indigenous knowledge research attempts to facilitate some communication along the continuum and beyond to policymakers, and so on.

The distinction between indigenous and scientific knowledge has an unpleasant political edge, with connotations of superiority and inferiority (Agrawal 1995). What is made of the apparent differences differs with views of development. The transfer of technology approach emphasizes differences; whereas the participatory approach downplays them, encouraging collaboration. The continuum seeks to overcome the pernicious side of the "we" and "them" divide by uniting us all. We need to beware of privileging one tradition above another in development contexts. The contributors to this volume believe that the 'stronger' scientific view should not dominate the 'weaker' local one, nor vice versa. While arguing for a more prominent place for local knowledge, we acknowledge that scientific knowledge under- pins technological advances, the dissemination of which, to reduce poverty, comprises much development. In advocating that we draw on the strengths of both perspectives, we acknowledge that conflict is inherent because sometimes their aims and values are not readily reconcilable. The objective is equitable negotiation, a central tenet of participatory development. The negotiations may be complex but development initiatives are more likely to be locally appropriate and sustainable.

A problem with the continuum model is that with its two ends it invites hierarchical discrimination between actors dotted along it, when in reality their positions may overlap, although their knowledge differs (e.g. ethnographers and national scientists). It is also static, with no sense of the dynamism that characterizes knowledge. In pondering these issues, I decided that the circle, with no separate extremities, is perhaps more inclusive, everything being linked together, as with no beginning or end it shows the mutuality of all knowledge and subverts notions of hierarchy (see Sillitoe 2002 of which the following is a summary statement). We also have a sense of movement, as science may cycle to IK, and equally IK to science, underlining the contemporary, syncretic character of IK (Figure 2).

The circle may be an advance but the potential for hierarchical thinking remains, with two poles customarily seen as top and bottom. It also suggests that some persons know less than

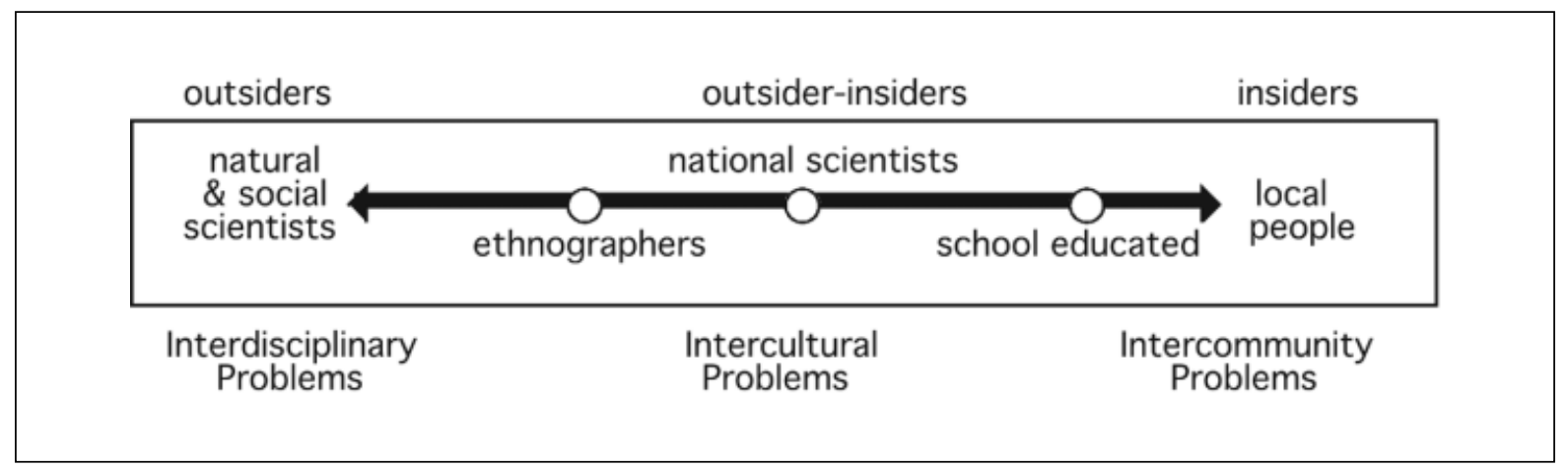

Figure 1: The knowledge continuum 
Figure 2: The circle model of science and indigenousknowledge relations

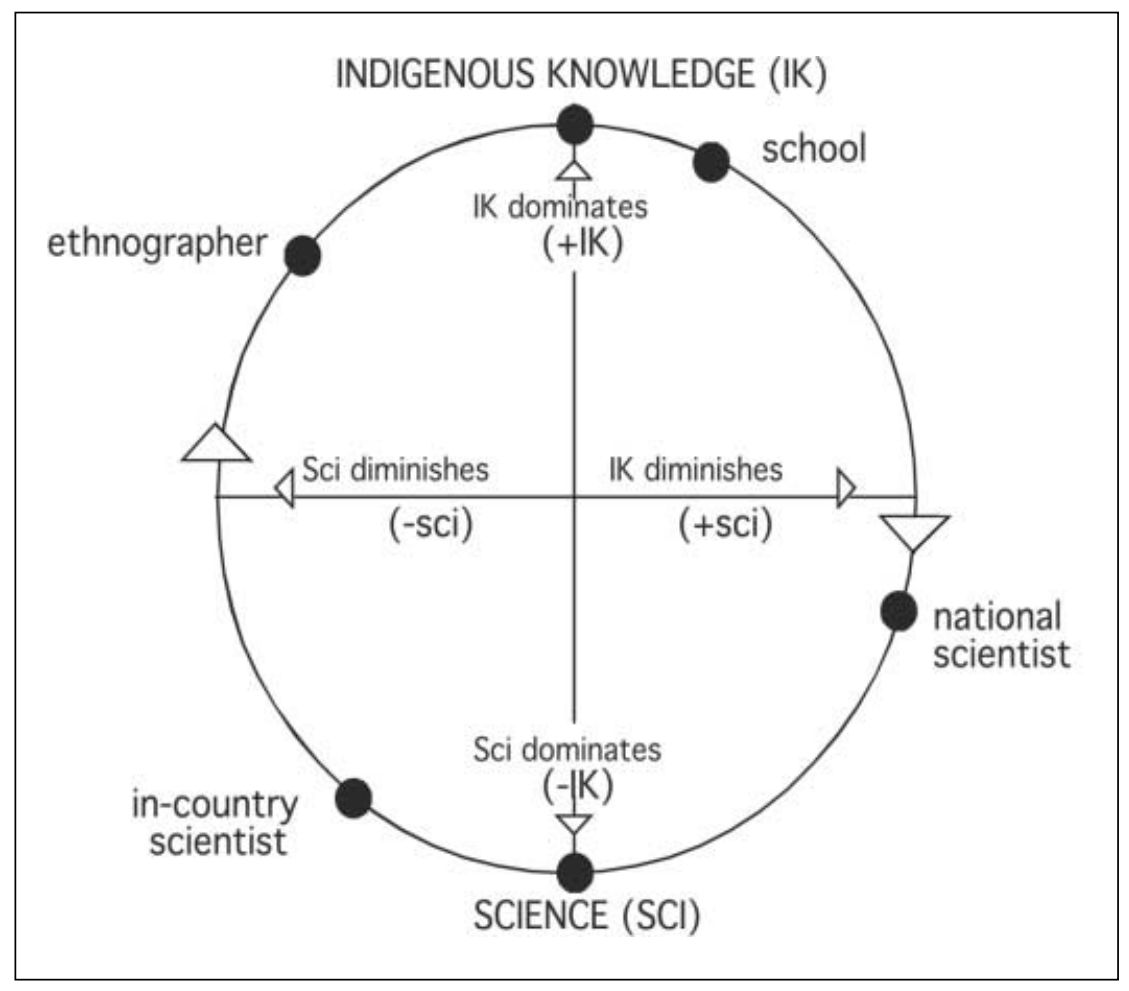

others about their fields (e.g. a national scientist knows less science than a visiting, foreign one situated at the science pole). It also continues to represent the indigenous and scientific as two monolithic knowledge traditions, when they comprise many strands (e.g. the knowledge of a soil physicist differs from that of a pest controller, and the knowledge held by members of any local community varies).

Realizing this I have suggested that we think in terms of meridians on a globe, each representing a different domain of knowledge, which allow us not only to cover variations in knowledge according to disciplines at the science pole and life experience at the indigenous one, but also to accommodate any cultural domain (e.g. according to religion, politics, economics etc.), complying with anthropological holistic demands. We do not have to envisage the meridians arranged like lines of longitude, all crossing at the same two polar points. We can arrange them randomly about the globe, obviating any tendency to depict knowledge hierarchically. The globe represents an interaction domain, which may be variously defined (e.g. geographically [e.g. as a local community], institutionally [e.g. as a development agency] or academically [e.g. agricultural science]). We can plot within it the positions of individuals who interact within the domain, according to their knowledge of meridian issues. The meridians act to pull them into a certain location, in effect locating individuals for a range of continua, in their knowledge/ culture cluster (Figure 3).

Persons interact in more than one global domain and we have to visualize linking several together (e.g. scientists visiting communities overseas come from institutions elsewhere). We have to work multidimensionally to represent the resulting complex galaxy networks, with many globes potentially interacting with one another. The complexity increases further when we consider that no individual or cultural constellation remains static over time. The multidimensional connections between domains are not rigid but constantly changing sets of relations. We have to accommodate such dynamism in seeking to incorporate IK research into devel- 


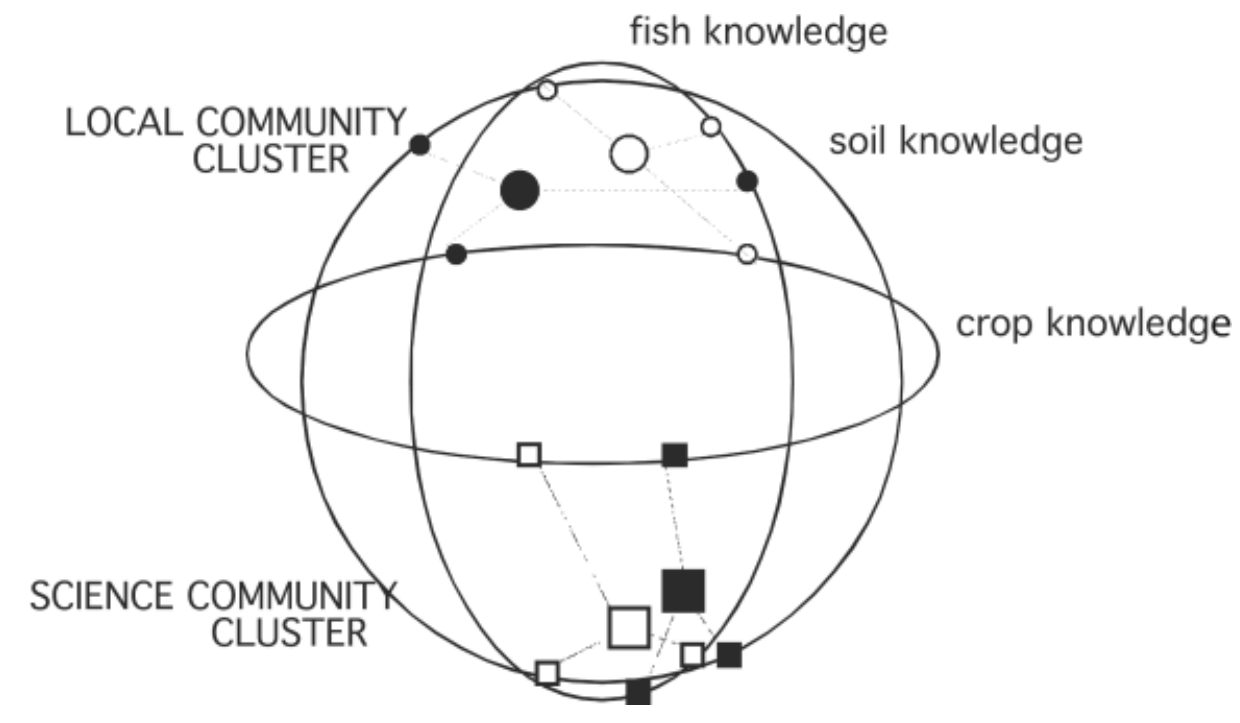

Figure 3: The globe model of science and indigenous-knowledge relations

opment. The aim of development after all is to promote such change, dramatically modifying IK with scientific perspectives. This model is currently no more than an intellectual construct which, while it may help us to conceptualize relations between knowledge traditions, will demand considerable work to make it useful. This is necessary if we are to use it in development contexts that demand practical action and not mere reflection. It will demand sophisticated computer modelling, something that the AID group is exploring through an e-science initiative.

In their contribution to this special issue, Stephen Lyon and Michael Fischer take up the issue of computer modelling, addressing it in the context of population displacement following natural disasters and pointing out how long-range planning to manage disasters will benefit from anthropological insights. They argue for formal modelling of cultural systems using the multi-agent approach and artificial intelligence to promote understanding of how disparate communities might co-exist in the event of natural disasters. After briefly outlining the culture concept and anthropology of communication, they turn to what computer modellers call 'ontologies' to show how anthropological understandings of such displaced communities can further dynamic modelling of their sociocultural systems, such that authorities might contribute to, rather than undermine, their resilience. They take the example of izzat or honour in South Asia to demonstrate the workings of the ontology idea in a cultural system.

\section{Justifying IK in International Development}

The following are some of the points made in arguing for the incorporation of IK in development. The contributions to this special issue of Anthropology in Action illustrate several of these points ethnographically.

- Indigenous knowledge enriches understanding of development opportunities 
within a cultural context, promoting culturally appropriate interventions relevant to people's needs;

(a) Projects, by understanding and valuing people's (indigenous) knowledge, can acquire a better understanding of the local position.

(b) Appreciation of local ideas and practices encourages more sustainable development interventions.

(c) Indigenous knowledge helps avoid expensive mistakes by preventing research and development initiatives that start from false premises through failing to show respect for local views.

- Indigenous knowledge facilitates communication across the interface between development project and local community, raising awareness of scope of research and possible development alternatives.

(a) It promotes identification of jointly perceived problems and negotiated action.

(b) It allows others to teach us, and discourages imposition of inappropriate foreign ideas.

(c) It helps reconcile different and potentially conflicting cultural perspectives regarding development initiatives by promoting awareness of others' views.

- Indigenous knowledge realizes the comparative advantages of both insider and outsider knowledge, creating opportunities for synergy.

(a) It conveys local knowledge to technicians/scientists such that they can appreciate its relevance and links communities more effectively with hightech scientific research / development. (b) It recognises that science may complement local understanding where it is inadequate; some interpret IK enquiry narrowly as identifying such knowledge gaps and targeting scientific research and extension to fill them. (c) It facilitates interdisciplinary, participatory research, recognized as necessary in development contexts to overcome narrow sector biases, IK being interdisciplinary by definition.

- Indigenous knowledge furthers empowerment of the poor through respect for their knowledge and management practices.

(a) It encourages people to have a voice and discourages unwanted interference in their lives.

(b) It promotes more effective local participation in planning, decision-making and so on.

(c) It may advance scientific understanding, as it may reveal intelligence unknown to science, and even challenge it.

- Indigenous knowledge advances awareness of local social issues.

(a) It helps assess the social impact of development interventions, notably relations between different interest groups such as the wealthy and the poor.

(b) It seeks to assess local variation in knowledge (by gender, age, class, occupation etc.) and accommodate different perspectives and agendas.

(c) It predicts possible conflict due to different interest groups manipulating interventions to their advantage.

- Indigenous knowledge informs extension with a local view, encouraging information flow towards locals.

(a) It helps tailor technical messages to problems as perceived locally, so that people can make more informed decisions.

(b) It encourages uptake by addressing problems from the local perspective.

(c) It helps inform people of the consequences of various choices.

Echoing the above contribution by Lyon and Fischer on managing natural disasters, Mariella Marzano explores in her article how farmers manage resources in a region of eastern Sri 
Lanka, with a particular focus on how they cope with environmental, notably climatic, perturbations. If global climate change proceeds as many scientists now predict, to what extent will these farmers' current knowledge and practices be able to cope? They will doubtless be dependent on their local heritage to a considerable measure and the authorities need to know what this comprises and people's possible capacity to manage before predicted climate change impact on their regions. Global warming is likely to negate interventions aimed at alleviating poverty. It is a topic of planned future AID research. While local communities are well placed to contribute to finding viable solutions, already responding to the effects of climatic variations in their regions, little knowledge of their experience reaches mainstream discourse on climate change (Rojas Blanco 2006). There is a pressing need for better two-way communication between policy discussions on climate change and poverty alleviation, and local experiences of coping with ever-changing environments (Adger et al. 2003).

\section{Some Methodological Issues}

Indigenous knowledge work is not straightforward. Several problems surround it, demanding attention to integrate IK into the development process. We need to formulate strategies that meet the development's demands-costeffective, time-effective, generating relevant insights, readily intelligible to non-experts etc.while not downplaying the difficulties so as to render the work effectively valueless. Much of the work done by members of the AID group seeks to address these generic methodological issues. (See Sillitoe et al. (2005) for a detailed discussion of IK methodological issues.)

A key issue is facilitating meaningful communication between development staff and local people, informing 'outsiders' about IK and 'insiders' about what scientific technology offers, so that both can better understand the alternatives and realize their comparative advantages. The promotion of more effective participation in the identification and tackling of problems can only be achieved so far as awareness and socio-political barriers will allow. We need a methodology that allows both 'outsiders' and 'insiders' to contribute as necessary, balancing between technocrats defining problems/constraints, which is arrogant and ethnocentric, and the local people doing so, which hits cultural barriers that thwart scientific research.

It is necessary to promote a collaborative atmosphere in which neither scientific nor local interests feel threatened, all parties having a role in negotiations, contributing vital skills and knowledge. The absence of a coherent IK approach that might interface effectively with science and technology is a limitation. The presentation of IK in a manner accessible to others, such that they can see its relevance to their work, means avoiding jargon-loaded and obscure accounts, while not overlooking insights gained in cross-cultural research, often in subtle arguments. There is a need to avoid oversimplification of complex issues, inviting distortion and misrepresentation in the search for user-friendly accounts.

The advancement of interdisciplinary work is central to IK research, particularly combining the technical know-how of natural scientists with the cultural empathy of social scientists (Sillitoe 2004). An integrated perspective requires learning from other disciplines, in addition to local people. There must be a genuine two-way flow of ideas and information between all parties. Motivation depends in considerable measure on fostering consensus decisions, joint ownership and open debate.

We cannot understand cultures by looking at individual parts in isolation: as complex systems, they manifest emergent properties that we can only see when all the parts are working together, and IK research seeks to introduce socio-cultural perspective into the narrowly focused work of technical specialists. It is not 
possible to predict which cultural domains might relate intimately to others; often unexpected practices impinge on one another. But we need to beware of accumulating ethnographic information not directly related to development issues, and even potentially disempowering people by representing their knowledge in inaccessible ways beyond their control, maybe infringing their intellectual property rights.

The 'one-offness' of IK-small-scale, culturally specific and geographically local-hampers its incorporation in development, impeding the formulation of generalizations that might inform wider policy and practice. It is necessary to evolve principles that will facilitate reliable generalization, going beyond case studies that are not cost effective to replicate. Alternatively, the advancement of a standard approach may be a red herring, considering the variety of knowledge traditions worldwide, their internal variations and their constant revision over time. This makes generalization potentially dangerous, imputing ideas elsewhere that may be inappropriate.

The dynamism of IK presents difficulties. Indigenous knowledge cannot be documented 'once and for all', as it is neither static nor uniform. It is subject to continual negotiation between stakeholders. We need an iterative strategy, closely linking development interventions to ongoing IK investigations.

The time scale required for ethnographic research is normally lengthy. It can take several years, not months or weeks, for someone unacquainted with a region to achieve meaningful insight into local knowledge and practices, and from this perspective inform development. This presents problems in development contexts with politically driven short-term demands for quick results. The understanding that can be accomplished in a single project will be of a different order to that achieved in long term ethnographic research. While some IK research may be attempted in short time frames, this normally involves compromises. It is a question not just of the time it takes to learn language, cultural repertoire, social scenario and so on, but also of the investment needed to win the trust and confidence of people who frequently have reason to be extremely suspicious of foreigners and their intentions.

At first sight IK work seems straightforward enough; we just have to ask some local culture bearers about their views of issues of development interest. But we soon encounter crosscultural issues that challenge what we think we know. Sympathetically accessing local concepts, and conveying something about them, is contentious. Knowledge is diffuse and communicated piecemeal in everyday life. It is not homogenous; there is often no consensus among the 'natives'. People transfer much through practical experience, and are unfamiliar with expressing all that they know in words. They may also carry knowledge, and pass it between generations, using alien idioms featuring symbols, myths, rites and so on. Translating what we hear into foreign words and concepts further misconstrues whatever it is that we manage to comprehend about another's views and actions, as the postmodern critique affirms (Fabian 2002, Sidky 2003). Understanding is inevitably limited given our outsider perspective, as in any ethnographic enquiry.

\section{The AID Research Group}

Staff associated with the AID Group are committed to advancing the life chances of the very poor by contributing to international development work (http://www.dur.ac.uk/ anthropology/research/aid/). We focus on participatory approaches and empowerment through advancing the indigenous or local knowledge in development agenda. We are building on a long tradition. The Anthropology Department in Durham has a history of promoting anthropology in development, with such distinguished scholars as Norman Long, Lucy Mair and Philip Mayer associated with it. We 
think that the discipline of anthropology is well suited to furthering local-level work of the kind that is now burgeoning in development, notably with the advent of participatory approaches. Anthropology has, after all, long recognized the significance of IK to furthering development interventions. It is the intellectual home of the ethnographic approach that underpins such work. As anthropologists we are trained to investigate cultural phenomena in depth, skilled to research social issues and view people in sociocultural context, and able to focus on problems relating to poverty, gender and so on.

The contribution to this issue of Aneesa Kassam and Alemayehu Lalise illustrates these points well in their discussion of the emotive issue of female circumcision in parts of Africa. It has long puzzled me, perhaps reflecting a certain ethnocentricity, how parents could allow their children to endure the painful operations often associated with initiation, particularly in the New Guinea context where they can be brutal. The article by Kassam and Lalise illustrates the strong social pressures on people to conform and ensure the marriageability of their daughters. Until recently, attempts to stop the practice of female genital cutting in Africa have failed to change behaviour significantly. The authors describe how an indigenous NGO has recently succeeded in reforming the practice in the Oromia region of Ethiopia, by facilitating discussion in communities using participatory methods, informing people of the detrimental health consequences of the practice and, critically, involving elders in debates about abandoning it. Its success compares with a few other programmes undertaken using similar strategies. The authors argue that these interventions have led to enduring change because they were made with a full understanding of the cultural context of the practice they sought to transform, a nice demonstration of a key tenet of the IK approach to development. Furthermore, the success of these programmes shows what can be achieved by empowering communities to direct the process of change from within.
Nongovernmental Organizations (NGOs) play a notable part in the promotion of local knowledge in development contexts. The IK networks, initially coordinated by the Indigenous Knowledge and Development Monitor and IK Worldwide and now functioning under several regional umbrellas, actively seek to promote local knowledge, as opposed to incorporating it by and by in development programmes as largely occurs when local NGOs co-operate with agencies. Those NGOs rooted in the local community are particularly aware of the localknowledge issues, although such grass-roots bodies have scarcely any influence. It is the national, regional and international organizations that have more clout but, as Manzurul Mannan argues in his contribution to this issue, they identify more with the policy aims of outside agencies in their bid to secure support and resources. They manipulate local interests to comply with what they think are the priorities of international funding agencies rather than represent these interests, particularly if they think they may jeopardize their chances. Drawing on his extensive experience of working with the Bangladesh Rural Advancement Committee (BRAC), one of the largest and most successful NGOs in the world, Mannan shows how the resulting conflicts of interests militate against not only the incorporation of local knowledge into development initiatives but also the subversion of research itself.

The AID Group is well placed to undertake work in IK and related efforts to promote grassroots participation. It has experience of working with NGOs, helping, for instance, to establish the Bangladesh Resource Centre for Indigenous Knowledge (Sen et al. 2000), part of the South Asian network of NGOs that seeks to promote local knowledge in development. The interdisciplinary nature of anthropology further makes it suitable for promoting integrated and systems approaches to rural development. The AID Group, for example, not only brings together a considerable range of disciplinary skills and experience able to address a wide range of 
development issues, but also collaborates regularly with colleagues in a range of other Departments, both in Durham and elsewhere. It is part of the Developing Areas Research Network in northeast England. The group has experience of short- and long-term research projects or consultancy work, and expertise in the design and management of research development projects. It also has experience of monitoring and evaluating missions, and, of course, training and education. We can offer training in IK work in development, ethnographic research methods, social research methodologies and participatory approaches to development. Staff have accepted visiting teaching appointments to strengthen institutions involved in development work and assisted in relevant curricula advancement.

Another issue where the AID Group has practice in assisting the understanding of local peoples' views concerns the arrival of largescale primary industry in their regions, such as mineral mining and commercial logging. In her contribution to this issue, Emma Gilberthorpe discusses problems of communication between Fasu speakers and the oil company piping crude from their territory in the southern highlands of Papua New Guinea. She has experimented with video to facilitate better understanding of issues by both sides. And there is also the looming issue of what happens when oil extraction stops and the royalty cheques cease to arrive for a population that has become used to them, the younger generations unfamiliar with previous subsistence practices of sago extraction, shifting cultivation, and hunting and fishing. We have worked on the converse of extractive industry too, on conservation and biodiversity projects, some staff having knowledge of the problems that attend the establishment of nature reserves/parks, when local landowners are excluded from parts of their territory. We have experience of land rights issues and associated litigation, and an ongoing interest in intellectual property rights issues which are increasingly prominent in development contexts, high- lighted by the empowerment agenda of participatory approaches.

The AID Group, in short, undertakes a range of anthropologically informed development work featuring innovative participatory approaches related chiefly to IK research and related efforts to promote grass-roots participation. We are particularly well qualified to pursue research involving a wide array of methodologies from in-depth, ethnographic, community-level studies to various social survey techniques, both qualitative and quantitative, including Participatory Rural Appraisal (PRA) and Rapid Rural Appraisal (RRA). The Durham Anthropology Department is broad based, covering both the sociocultural and biological sides of the discipline, and members of the group contribute to research and development on livelihood and health in a sociocultural and biological context, which covers the sustainable management of natural resources (agriculture, fisheries, forestry etc.) and the improvement of health and well-being (disease, nutrition, sanitation). Our experience particularly qualifies us to assist in work on the environment, as it is broadly defined. Regionally, we have almost global coverage with staff working extensively in Africa, South and Southeast Asia, Europe, South America and the Pacific.

\section{Acknowledgement}

I presented a version of this article at Newcastle University as the keynote address to the Developing Areas Research Network meeting on 'Indigenous Knowledge and Development' and I am grateful to participants for their helpful comments.

Paul Sillitoe is Professor in the Department of Anthropology at the University of Durham. Research interests include natural resources management, appropriate technology, and development, both sustainability and cultural aspects and changing relation to the social order. Other areas of interest 
are economics, politics, social and environmental change, conservation, land issues, human ecology and ethnoscience. He has long-standing interests in the Pacific (Papua New Guinea) and South Asia (Bangladesh). (Paul Sillitoe, Department of Anthropology, University of Durham, 43 Old Elvet, DURHAM DH1 3HN, UK. E-mail: paul.sillitoe@durham.ac.uk.)

\section{References}

Adger, W.N., A. Huq, K. Brown, D. Conway and M. Hulme 2003. 'Adaptation to Climate Change in the Developing World', Progress in Development Studies 3 (3): 179-195.

Agrawal, A. 1995. 'Dismantling the Divide Between Indigenous and Scientific Knowledge', Development and Change 26: 413-439.

Antweiler, C. 1998. 'Local Knowledge and Local Knowing: an Anthropological Analysis of Contested "Cultural Products" in the Context of Development', Anthropos 93: 469-494.

Bentley, J.W. and P.S. Baker 2005. 'Understanding and Getting the Most from Farmers' Local Knowledge', in J. Gonsalves, T. Becker, A. Braun, D. Campilan, H. Chavez, E. Fajber, M. Kapiriri, J. Rivaca-Caminade and R. Vernooy (eds) Participatory Research and Development for Sustainable Agriculture and Natural Resource Management: a Sourcebook, CIP-UPWARD/IDRC.

Brokensha, D., D.M. Warren and O. Werner 1980 (eds). Indigenous Knowledge Systems and Development, Lanham: University Press of America.

Chambers, R., A. Pacey and L.A. Thrupp 1989 (eds). Farmers First: Farmer Innovation and Agricultural Research, London: Intermediate Technology Publications Limited.

Cooke, B. and U. Kothari 2001 (eds). Participation: The New Tyranny, London: Zed Books.

DeWalt, B.R. 1994. 'Using Indigenous Knowledge to Improve Agriculture and Natural Resource Management', Human Organisation 53 (2): 123-131.

Ellen, R., P. Parkes and A. Bicker 2000 (eds). Indigenous Environmental Knowledge and Its Transformations, Amsterdam: Harwood Academic.

Fabian, J. 2002. Time and The Other, New York: Columbia University Press.

FAO 1989. Farming Systems Development, Rome: FAO.
Howes, M. and R. Chambers 1979. 'Indigenous Technical Knowledge: Analysis Implications and Issues', Institute of Development Studies Bulletin 10 (2): 5-11.

Kloppenburg, J. 1991. 'Social Theory and the De/construction of Agricultural Science: Local Knowledge for an Alternative Agriculture', Rural Sociology 56 (4): 519-548.

Purcell, T.W. 1998. 'Indigenous Knowledge and Applied Anthropology: Questions of Definition and Direction', Human Organization 57 (3): 258-272.

Rhoades, R. and R. Booth 1982. 'Farmer-Back-toFarmer: A Model for Generating Acceptable Agricultural Technology', Agricultural Administration 11: 127-137.

Richards, P. 1985. Indigenous Agricultural Revolution, London: Hutchinson.

Rojas Blanco, A.V. 2006. 'Local Initiatives and Adaptation to Climate Change', Disasters 30 (1): 140-147.

Sen, S., B. Angell and A. Miles 2000. 'The Bangladesh Resource Centre for Indigenous Knowledge and Its Network', in P. Sillitoe (ed.) Indigenous Knowledge Development in Bangladesh: Present and Future, London: Intermediate Technology Publications, 213-218.

Shepherd, C.J. 2004. 'Agricultural Hybridity and the "Pathology" of Traditional Ways: The Translation of Desire and Need in Postcolonial Development', Journal of Latin American Anthropology 9 (2): 235-266.

Sidky, H. 2003. A Critique of Postmodern Anthropology, Lewiston: Edwin Mellen Press.

Sillitoe, P. 1998a. 'The Development of Indigenous Knowledge: A New Applied Anthropology', Current Anthropology 39 (2): 223-252.

Sillitoe, P. 1998b. 'What Know Natives?: Local Knowledge in Development', Social Anthropology 6 (2): 203-220.

Sillitoe, P. 2002. 'Globalizing Indigenous Knowledge', in P. Sillitoe, A. Bicker and J. Pottier (eds) Participating in Development: Approaches to Indigenous Knowledge, London: Routledge (Association of Social Anthropologists' Monograph Series No. 39), 108-138.

Sillitoe, P. 2004. 'Interdisciplinary Experiences: Working With Indigenous Knowledge in Development', Interdisciplinary Science Reviews 29 (1): 6-23.

Sillitoe, P., P. Dixon and J. Barr 2005. Indigenous Knowledge Inquiries: A Methodologies Manual For Development, London: Intermediate Technology Publications. 
Thrupp, L.A. 1989. 'Legitimizing Local Knowledge: From Displacement to Empowerment for Third World People', Agriculture and Human Values 3: 13-25.

Warren, D.M. 1991. Using Indigenous Knowledge in Agricultural Development, World Bank Discussion Papers, No. 127. Washington, D.C.
Warren, D.M., L. Slikerveer and D. Brokensha 1995 (eds). The Cultural Dimension of Development: Indigenous Knowledge Systems, London: Intermediate Technology Publications.

Wright, S. and S. Nelson. 1995 (eds). Power and Participatory Development: Theory and Practice, London: Intermediate Technology Publications. 